\title{
Thermal-Diffusivity Dependence on Temperature of Gadolinium Calcium Oxoborate Single Crystals
}

\author{
D. Trefon-Radziejewska $\cdot$ J. Bodzenta . \\ T. Łukasiewicz
}

Received: 14 October 2011 / Accepted: 31 March 2012 / Published online: 20 April 2012

C The Author(s) 2012. This article is published with open access at Springerlink.com

\begin{abstract}
Thermal diffusivities of pure and doped gadolinium calcium oxoborate (GdCOB) single crystals were measured as a function of the temperature along optical indicatrix axes $X, Y$, and $Z$. Three GdCOB samples were investigated, chemically pure single crystal, the one doped with 4 at $\%$ of $\mathrm{Nd}$ and the next one doped with 7 at $\%$ of $\mathrm{Yb}$. Measurements were carried out for temperature range $40{ }^{\circ} \mathrm{C}$ to $300{ }^{\circ} \mathrm{C}$. Determination of the thermal diffusivity based on an analysis of thermal wave propagation in the sample. For a detection of temperature disturbance propagating in the sample the mirage effect was used. Obtained results show that the thermal diffusivity decreases with the increase of sample temperature for all investigated crystals. The GdCOB single crystals reveal a strong anisotropy. The thermal diffusivity along $Y$ direction has the highest value while values obtained in $X$ and $Z$ axes are much lower. Dopants cause decrease in the thermal diffusivity for all investigated directions.
\end{abstract}

Keywords Nonlinear optical crystals · Temperature dependence . Thermal diffusivity $\cdot$ Thermal wave measurement

\section{Introduction}

In the last decade demand for application of nonlinear optical materials in laser systems has been increasing. Among a wide group of non-linear optical crystals, gadolinium calcium oxoborate $\mathrm{GdCa}_{4} \mathrm{O}\left(\mathrm{BO}_{3}\right)_{3}(\mathrm{GdCOB})$ has gained much attention. $\mathrm{GdCOB}$ has

D. Trefon-Radziejewska $(\varangle) \cdot$ J. Bodzenta

Institute of Physics, Silesian University of Technology, Krzywoustego 2, 44-100 Gliwice, Poland

e-mail: Dominika.Trefon@polsl.pl

T. Łukasiewicz

Institute of Electronic Materials Technology, Wólczyńska 133, 01-919 Warsaw, Poland 
a monoclinic biaxial crystal structure and belongs to the $\mathrm{Cm}$ space group. The unit cell parameters are: $a=8.099 \AA, b=16.031 \AA, c=3.556 \AA, \alpha=\gamma=90^{\circ}$, and $\beta=101.242^{\circ}[1]$.

Pure and rare-earth ion-doped GdCOB crystals possess many interesting physical properties such as a high-nonlinear coefficient, high-damage threshold, high-optical gain at $1.06 \mu \mathrm{m}$, and low self-absorption at the second-harmonic wavelength [2]. They find applications as efficient crystals for frequency conversion and self-frequency doubling laser [3]. Optical nonlinearity makes them also interesting as materials for color displays, high-density optical data storage devices, laser printers, biotechnology, and eye-safe light generation [4-6].

Considering application of these crystals in laser techniques, it is crucial to investigate their thermal properties. They influence the maximum laser output power, the quality of a laser beam, and laser efficiency [7]. High-thermal diffusivity insures good heat abstraction during laser action. Because laser systems based on these crystals typically work at elevated temperatures, the dependence of the thermal properties on temperature should be known. Results from our work [8] showed that the thermal diffusivity of GdCOB is much lower than that of yttrium-aluminum garnet and yttrium orthovanadate crystals. GdCOB crystals also exhibit strong anisotropy of the thermal diffusivity connected with their crystallographic structures. Moreover, results reveal that dopants introduced into a crystal lattice diminish the thermal diffusivity [8-10]. However, a systematic investigation of the thermal-diffusivity dependence on temperature of GdCOB single crystal, taking into account doping and the anisotropy has not been done.

This article presents the results of investigations of the thermal-diffusivity dependence on temperature for pure, 4 at $\%$ of Nd-doped, and 7 at $\%$ of Yb-doped GdCOB single crystals. The thermal diffusivity was measured along optical indicatrix axes $X, Y$, and $Z$. In the next section, the samples and measuring method used for the investigation are described. Results of measurements carried out in the temperature range from $40^{\circ} \mathrm{C}$ to $300^{\circ} \mathrm{C}$ are reported in the Sect. 3. Final conclusions end the paper.

\section{Measurements}

\subsection{Samples}

Three GdCOB samples were examined: pure, doped with 4.0 at $\%$ of $\mathrm{Nd}^{3+}$, and doped with 7.0 at $\%$ of $\mathrm{Yb}^{3+}$. All crystals were grown by the Czochralski pulling method in the Institute of Technology of Electronic Materials (ITME) in Warsaw. Details related to the growing process were described elsewhere [9]. Samples were cut into rectangular prisms with faces perpendicular to the principal axes of the optical indicatrix. Basic information about samples is presented in Table 1.

\subsection{Measuring Method}

The thermal wave method with mirage detection has been used for thermal diffusivity measurements. The experiment is based on the one-dimensional model 
Table 1 Basic information about samples

\begin{tabular}{llllll}
\hline Sample & & Dopant & \multicolumn{3}{l}{ Dimension $\left(10^{-3} \mathrm{~m}\right)$} \\
\cline { 5 - 6 } & & & {$[X]$} & {$[Y]$} & {$[Z]$} \\
\hline$\# 1$ & $\mathrm{GdCa}_{4} \mathrm{O}\left(\mathrm{BO}_{3}\right)_{3}$ & - & 9.98 & 8.16 & 9.21 \\
$\# 2$ & $\mathrm{GdCa}_{4} \mathrm{O}\left(\mathrm{BO}_{3}\right)_{3}$ & 4.0 at\% of $\mathrm{Nd}$ & 10.01 & 8.32 & 9.21 \\
$\# 3$ & $\mathrm{GdCa}$ & 7.0 at\% of $\mathrm{Yb}$ & 9.95 & 8.36 & 9.20 \\
\hline
\end{tabular}

of heat propagation in a thermally thick sample. In this method, the phase delay of a thermal wave propagating in the sample as a function of distance $h$ from its source is investigated. This dependence can be described by [11]

$$
\Delta \varphi=\sqrt{\frac{\pi f}{\alpha}} h+\varphi_{0}
$$

where $f$ is the thermal wave frequency and $\varphi_{0}$ is a constant. In our experiments the phase delay was determined from a measurement of the time delay $\Delta t$ of the temperature disturbance at a distance $h$ in relation to the harmonic component of the heater temperature. The relation between $\Delta \varphi$ and $\Delta t$ is

$$
\Delta \varphi=2 \pi f \Delta t
$$

The thermal diffusivity was evaluated from fitting the experimental data with a straight line. In the experiment described in this paper, the modulation frequency was $100 \mathrm{mHz}$. The thermal-diffusivity measurements were carried out for the range of temperature from $40{ }^{\circ} \mathrm{C}$ to $300{ }^{\circ} \mathrm{C}$.

\subsection{Experimental Setup}

To determine the thermal diffusivity of a sample for a particular temperature, an original experimental setup was used. The investigated crystal was placed in a chamber between two resistance heaters, which allowed sample heating and insured the same temperature over the whole volume of the sample. For the purpose of the temperature disturbance generation, the current of the selected heater was modulated with a constant frequency. In experiments described in this paper, the modulation frequency was $100 \mathrm{mHz}$. In order to monitor the temperature of the upper and lower sample surfaces, a copper plate with thermocouples was placed between the sample and the heaters. To assure low heat resistance, thin layers of a heat conducting paste were applied at all contacts. A sample and heaters were in good thermal insulation as a result of evacuation of the chamber to a pressure of a few "Pa". At the beginning of each measurement, the temperature of the sample was stabilized at a set value. Then temperature modulation around the set point was applied. Detection of a temperature disturbance, which propagated in the sample, based on the mirage effect. A detecting light beam from a He-Ne laser (LASOS 7672) was passed through the sample. The deflection 


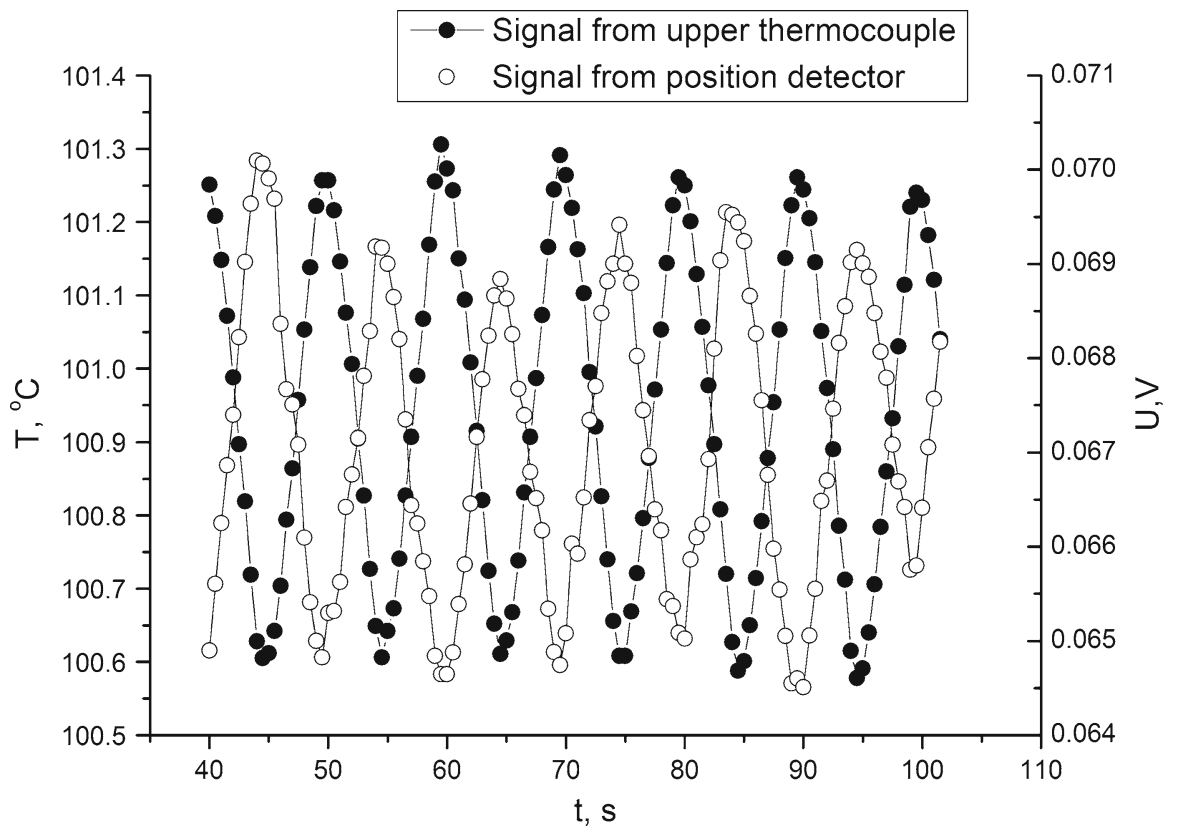

Fig. 1 Time delay of temperature disturbance propagating along the $X$ axis of 4 at $\%$ Nd:GdCOB sample. Signal from the position detector was measured at a selected distance of the probing beam from the modulated upper heater

of this beam was registered by a position sensor (Silicon Sensor DL400-7PCBA). The signals from the detector and from both thermocouples were fed to data acquisition unit (Agilent 34970A) and collected on a PC via an RS-232 interface. Exemplary time dependencies of signals from the thermocouple and the position detector registered for 4 at $\% \mathrm{Nd}$ :GdCOB and the thermal wave propagating along the $X$ axis are shown in Fig. 1.

\section{Results and Discussion}

Thermal diffusivities of pure and doped GdCOB crystals were determined for the directions of the optical indicatrix axes at seven temperatures in the range from $40{ }^{\circ} \mathrm{C}$ to $300{ }^{\circ} \mathrm{C}$. The thermal diffusivity was obtained by fitting a straight line to the $\Delta \varphi$ versus $h$ data. As an example, the dependence obtained for 4 at $\% \mathrm{Nd}$ :GdCOB in the $X$ direction at $100{ }^{\circ} \mathrm{C}$ is shown in Fig. 2. From the slope of the line fitted to the experimental points, the thermal diffusivity was calculated. The dependencies of the thermal diffusivity on the temperature determined for all investigated samples along the $X, Y$, and $Z$ axes are presented in Fig. 3. The obtained results confirm that GdCOB crystals exhibit strong anisotropy of the thermal diffusivity connected with their monoclinic structure. For pure and doped GdCOB crystals, the highest thermal diffusivities are observed along the $Y$ axis, and the lowest one along the $X$ axis. For all studied crystals the thermal diffusivity decreases when the temperature increases. 


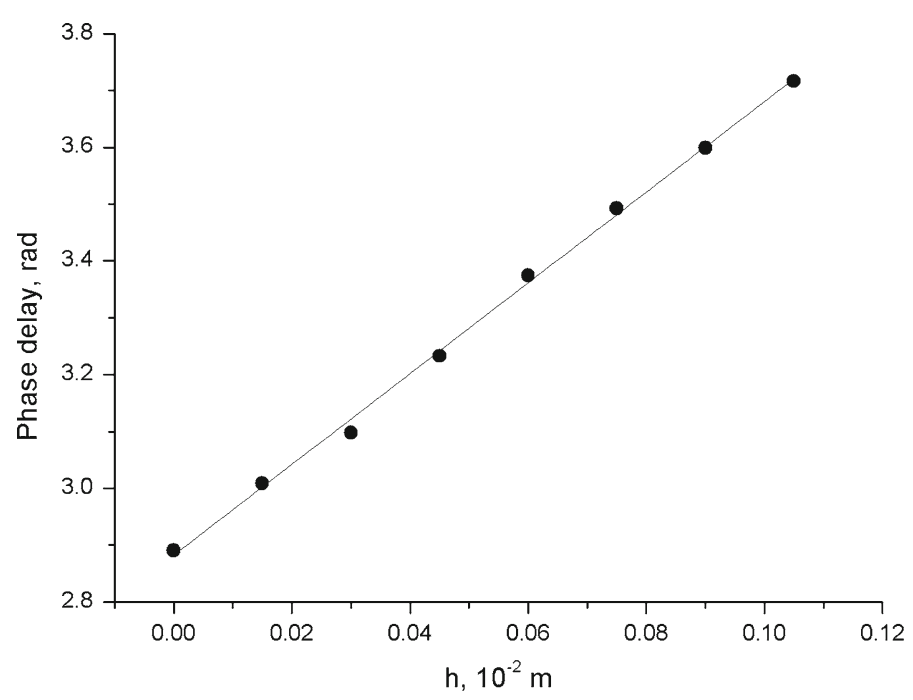

Fig. 2 Phase delay of the signal as a function of the distance $h$ from the sample surface measured in 4 at $\%$ $\mathrm{Nd}$ :GdCOB crystal for the $X$ axis at $100{ }^{\circ} \mathrm{C}$

It drops relatively fast near room temperature and becomes almost constant at about $250{ }^{\circ} \mathrm{C}$ to $300^{\circ} \mathrm{C}$. One can also notice that the temperature dependence of the thermal diffusivity is influenced by impurities. The absolute change of the thermal diffusivity in the investigated temperature range is much higher for pure crystals in comparison with doped GdCOB. Moreover, the impurities cause a considerable drop in the thermal diffusivity near room temperature while their influence at higher temperatures becomes less pronounced. Such a behavior can be explained by the change of the dominant phonon scattering mechanism with temperature. At lower temperatures the most important scattering mechanism is the scattering of phonons on defects. Impurities create additional scattering centers and limit the mean-free path of phonons and, as a consequence, the thermal diffusivity. At higher temperatures the phonon concentration increases and phonon-phonon scattering prevails. The influence of impurities is less pronounced, and differences between pure and doped samples become smaller. One can expect that at very high temperature all samples seem to exhibit very similar thermal properties, and the influence of dopants becomes negligible.

\section{Conclusion}

Thermal-diffusivity dependencies on temperature of a few GdCOB single crystals with different doping concentrations were investigated. The obtained results showed strong thermal anisotropy. Beginning from $40{ }^{\circ} \mathrm{C}$, the lowest values of the thermal diffusivity were obtained along the $X$ axis and was about $0.81 \times 10^{-6} \mathrm{~m}^{2} \cdot \mathrm{s}^{-1}$ for pure crystal, $0.59 \times 10^{-6} \mathrm{~m}^{2} \cdot \mathrm{s}^{-1}$ for Nd-doped crystal, and $0.72 \times 10^{-6} \mathrm{~m}^{2} \cdot \mathrm{s}^{-1}$ for Yb-doped crystal. The values obtained in the $Z$ direction were higher in comparison to the $X$ direction and equal to $1.22 \times 10^{-6} \mathrm{~m}^{2} \cdot \mathrm{s}^{-1}, 0.79 \times 10^{-6} \mathrm{~m}^{2} \cdot \mathrm{s}^{-1}$, 

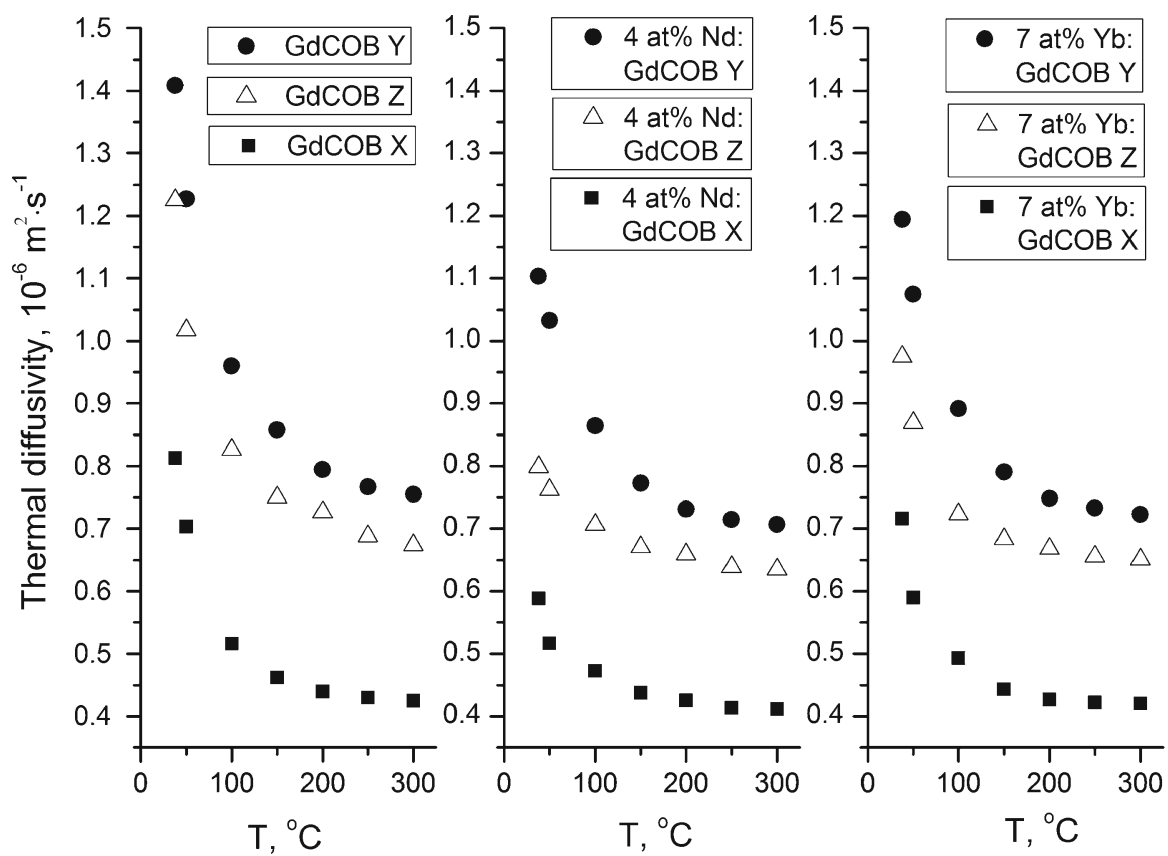

Fig. 3 Thermal-diffusivity dependence on temperature of pure GdCOB, doped with 4 at $\%$ of $\mathrm{Nd}$ and 7 at $\%$ of $\mathrm{Yb}$

and $0.97 \times 10^{-6} \mathrm{~m}^{2} \cdot \mathrm{s}^{-1}$, respectively. The highest values were measured in the $Y$ direction. They are $1.41 \times 10^{-6} \mathrm{~m}^{2} \cdot \mathrm{s}^{-1}$ for pure crystal, $1.10 \times 10^{-6} \mathrm{~m}^{2} \cdot \mathrm{s}^{-1}$ for Nd-doped crystal, and $1.19 \times 10^{-6} \mathrm{~m}^{2} \cdot \mathrm{s}^{-1}$ for Yb-doped crystal. It is observed that the doping significantly changes the thermal properties of GdCOB crystals near room temperature. The interesting fact is that a $4 \mathrm{at} \%$ addition of $\mathrm{Nd}$ has a stronger influence on the thermal diffusivity than a 7 at $\%$ addition of $\mathrm{Yb}$. It suggests that the scattering ratio depends not only on the dopant concentration, but also on the location of impurity ions in the lattice, which can be different for $\mathrm{Nd}$ and $\mathrm{Yb}$. All measured crystals show a decrease in thermal diffusivity with increasing temperature. The largest decrease in thermal diffusivity was observed for the pure sample (between $45 \%$ and $48 \%$ depending on the direction) in the $40{ }^{\circ} \mathrm{C}$ to $300{ }^{\circ} \mathrm{C}$ temperature range. Relative changes in the thermal diffusivity were smaller for GdCOB doped with Nd (between $20 \%$ and $30 \%$ ) than with $\mathrm{Yb}$ ions (30\% and $40 \%$ ) in the measured temperature range. The important fact is that at high temperatures thermal diffusivities of pure and doped crystals have very similar values for each investigated direction. So the influence of doping on the thermal diffusivity above a certain temperature can be neglected.

Open Access This article is distributed under the terms of the Creative Commons Attribution License which permits any use, distribution, and reproduction in any medium, provided the original author(s) and the source are credited. 


\section{References}

1. A. Brenier, A. Majchrowski, E. Michalski, T. Lukasiewicz, Opt. Commun. 217, 395 (2003)

2. M. Iwai, T. Kobayashi, H. Furuya, Y. Mori, T. Sasaki, Jpn. J. Appl. Phys. 36, 276 (1997)

3. S. Zhang, Z. Cheng, G. Zhou, Y. Sun, X. Hou, X. Liu, J. Han, Z. Shao, H. Chen, Prog. Cryst. Growth Charact. Mater. 40, 81 (2000)

4. G. Aka, E. Reino, P. Loiseau, D. Vivien, B. Ferrand, L. Fulbert, D. Pelenc, G. LucasLeclin, P. Georges, Opt. Mater. 26, 431 (2004)

5. T. Kubota, R. Takahashi, T.-W. Kim, T. Kawazoe, M. Ohtsu, N. Arai, M. Yoshimura, H. Nakao, H. Furuya, Y. Mori, T. Sasaki, Y. Matsumoto, H. Koinuma, Appl. Surf. Sci. 223, 241 (2004)

6. H. Nakao, M. Nishida, T. Shikida, H. Shimizu, H. Takeda, T. Shiosaki, J. Alloys Compd. 408, 582 (2006)

7. Y. Yu, Y. Cheng, H. Zhang, J. Wang, X. Cheng, H. Xia, Mater. Lett. 60, 1014 (2006)

8. J. Bodzenta, A. Kazmierczak-Bałata, J. Mazur, Cent. Eur. J. Phys. 8, 207 (2010)

9. J. Bodzenta, A. Kazmierczak-Balata, T. Lukasiewicz, B. Hofman, J. Kucytowski, Acta Phys. Pol. A 114, 27 (2008)

10. A. Kazmierczak-Bałata, J. Bodzenta, P. Szperlich, K. Wokulska, J. Kucytowski, T. Lukasiewicz, W. Hofman, J. Alloys Compd. 481, 622 (2009)

11. J. Bodzenta, M. Pyka, J. Phys. IV France 137, 259 (2006) 